\title{
https://doi.org/10.1007/s10357-020-3724-z \\ Diese andere Potenzielle Katastrophe: Wie kann der EGMR dazu beitragen, die Klimakrise einzudämmen?
}

\author{
Katharina Franziska Braig, Angela Ehlers-Hofherr*
}

(c) Springer-Verlag GmbH Deutschland, ein Teil von Springer Nature 2020

\begin{abstract}
In den letzten Jahren hat der EGMR zunehmend anerkannt, dass einzelne Konventionsgarantien, insbesondere Art. 2 und Art. 8 EMRK, dahingehend ausgelegt werden können, dass sie unter bestimmten Bedingungen den Einzelnen auch vor Umweltverschmutzungen schützen.

In diesem Artikel wird analysiert, inwieweit sich insbesondere die im Kontext des Umweltschutzes vom EGMR ausgearbeiteten positiven staatlichen Schutzpflichten auf den Kontext des Klimawandels übertragen lassen. Inwieweit können Einzelpersonen, NGOs und auch Unternehmen vor dem EGMR klimaschützende Maßnahmen vom Staat einfordern, wenn sie z. B. ihr Recht auf Eigentum oder ihr Recht auf Leben durch die negativen Auswirkungen des Klimawandels bedroht sehen? Neben den Chancen die die evolutive Rechtsprechung des EGMR in diesem Bereich bietet, geht der Artikel auch auf die Grenzen der positiven Schutzpflichten in diesem Zusammenhang sowie auf verschiedene Klimaklagen vor nationalen Gerichten ein, die hier möglicherweise richtungsweisend sein könnten.
\end{abstract}

Angesicht der Corona-Pandemie besteht die Gefahr, dass eine andere potenzielle Katastrophe, der Klimawandel, in den Hintergrund rückt: ein ebenso globales Problem, das - sollten wir nicht bald beginnen zu handeln - droht deutlich gefährlicher zu werden als Covid-19 es je sein könnte. Wenn Staaten nicht aus eigenem Antrieb ausreichend handeln, können Unternehmen, Privatpersonen und NGOs versuchen mehr staatliche Schutzmaßnahmen gegen die negativen Folgen des Klimawandels gerichtlich einzufordern.

Dieser Artikel eruiert, inwieweit Unternehmen, Privatpersonen und NGOs die Schutzmechanismen des Europäischen Gerichtshofs für Menschenrechte (EGMR) hier nutzen können. Denn es scheint bereits jetzt klar zu sein, dass Staaten, um z. B. die Grundrechte auf Leben, Gesundheit, Existenzminimum und Eigentum zu schützen, mindestens die im Pariser Klima-Abkommen vereinbarte Begrenzung der globalen Erwärmung auf 1,5 Grad gegenüber vorindustriellem Niveau einhalten müssen. ${ }^{2}$ Die Klimaproblematik könnte daher - so die ehemalige Vizepräsidentin des EGMR Angelika Nußberger kurz vor ihrem turnusmäßigen Ausscheiden Ende 2019 - unter Berufung auf die Grundrechte auch vor den EMGR gebracht werden. ${ }^{3}$

Im Folgenden möchten wir daher untersuchen, inwieweit die für den Menschen schädlichen Auswirkungen für den Klimawandel, z.B. vermehrte Waldbrände und Überschwemmungen, die unbestreitbar globale Auswirkungen auf die Wahrnehmung der Menschenrechte, insbesondere das Recht auf Leben ${ }^{4}$ haben, auch Gegenstand von Verfahren vor dem EGMR werden können.

Das Recht auf Leben zu schützen ist vornehmste Pflicht des EGMR, verankert in Art. 2 der Europäischen Menschenrechtskonvention (EMRK). Unstreitig ist davon auszugehen, dass sich der Klimawandel negativ auf die in der EMRK garantierten Rechte auswirken kann. Aber nicht jede negative Auswirkung auf ein essentielles europäisches Schutzgut führt $\mathrm{zu}$ einem effektiven gerichtlichen Schutz.

Dr. Katharina Franziska Braig, LL.M. (London), Brüssel, Belgien, Angela Ehlers-Hofherr, Rechtsanwältin und Mediatorin,

Kanzlei Sterr-Kölln \& Partner,

Freiburg i.B., Deutschland
Inwieweit kann also der EGMR uns tatsächlich vor den negativen Auswirkungen des Klimawandels schützen?

In diesem Artikel nähern wir uns der Antwort über die vom EGMR entwickelte Doktrin der positiven Verpflichtungen. Denn sie scheint uns am ehesten geeignet um Beschwerden über die negativen Auswirkungen des Klimawandels zu adressieren.

Obwohl die EMRK in erster Linie einen geeigneten Rahmen für territorial begrenzte Individualklagen bietet, kann der EGMR theoretisch auch Opfern globaler, im Umweltkontext auftretender Menschenrechtsverletzungen wirksame und praktische Schutzmaßnahmen bieten.

\section{Erste Klimaklagen auf nationaler Ebene}

In letzter Zeit hatten bereits mehrere nationale Gerichte die Gelegenheit, den Zusammenhang zwischen der EMRK, der umweltrelevanten Rechtsprechung des EGMR und der Klimaproblematik einzelner Staaten zu erörtern.

Im Fall Urgenda ${ }^{5}$ verklagte eine Bürgerplattform die Niederlande vor dem Bezirksgericht in Den Haag. Die Bürgerplattform hatte argumentiert, dass der niederländische Staat es versäumt hatte, ausreichende aktive Maßnahmen zu ergreifen um seine $\mathrm{CO}_{2}$-Emissionen zu senken. Im Juni 2015 entschied das Bezirksgericht, dass die Regierung ihre Treibhausgasemissionen bis Ende $2020 \mathrm{um}$ mindestens 25 Prozent senken muss (im Vergleich zu 1990). In dem Urteil wird die niederländische Regierung dazu aufgefordert, sofort wirksamer gegen den Klimawandel vorzugehen. In seinem Urteil verwies das Bezirksgericht ausführlich auf die Rechtsprechung des EGMR. Obwohl das Haager Bezirksgericht Art. 2 und 8 EMRK nicht direkt für anwendbar erklärte, da die Beschwerdeführer aus seiner Sicht nicht die dafür erforderliche Opfereigenschaft besaßen, folgerte er, dass die EMRK-Standards zur Beurteilung des staatlichen Ermessensspielraums sowie zur Beantwortung der Frage nach dem Mindestmaß an staatlicher Sorgfalt herangezogen werden könnten.

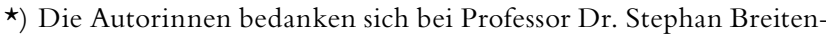
moser (Basel) für zahlreiche wertvolle Inputs und Denkanstöße ohne die dieser Artikel nicht entstanden wäre.

1) So gehen z.B. Wissenschaftler davon aus, dass durch die negativen Folgen des Klimawandels in den kommenden Jahren sehr viele Menschen allein an Hunger sterben werden, s.z.B. Springmann et al., ,Global and regional health effects of future food production under climate change: a modelling study', 2016, The Lancet 387.10031,1937-1946; hinzu kommen z. B. vermehrt Hitzetote, s.z.B. Nowak, ,Global warming - the German picture', Deutsches Ärzteblatt International, 2019, 116: 519-20, 10.3238/ arztebl.2019.0519.

2) Ekardt/Wieding/Zorn, ,Paris Agreement, Precautionary Principle and Human Rights: Zero Emissions in Two Decades?', Sustainability 10(8), 2018, 2812, https://doi.org/10.3390/su10082812.

3) „Klimaklagen werden laut Richterin künftig zunehmen“, Stand 22.3.2020, abrufbar unter https://www.zeit.de/gesellschaft/ 2019-12/europaeischer-gerichtshof-klimaklagen-zunahme.

4) The Office of the High Commissioner for Human Rights, Understanding Human Rights and Climate Change, submission the 21st Conference of Parties to the UNFCCC, 27.11.2015, 13-24

5) Rechtbank Den Haag, The District Court of the Hague, Urgenda C/09/456689, 13-1396, 24.5.2015, Stand 22.3.2020, abrufbar unter https://elaw.org/nl.urgenda.15. 
Im Oktober 2018 bestätigte das Berufungsgericht in Den Haag die Entscheidung des Bezirksgerichts dem Grunde nach und stellte fest, dass die Niederlande ihre Sorgfaltspflicht verletzen, indem sie es versäumen eine ehrgeizigere Verringerung der Treibhausgasemissionen anzustreben ,und stimmte der Feststellung der Vorinstanzen [dahingehend] zu, dass der Staat seine Emissionen bis Ende 2020 um mindestens 25 Prozent senken sollte." Es sei angemessen, in diesem Zusammenhang von einer ,,realen Bedrohung des gefährlichen Klimawandels zu sprechen, der das ernsthafte Risiko mit sich bringe, dass die gegenwärtige Generation von Bürgern mit dem Verlust von Leben und/ oder einer Störung des Familienlebens konfrontiert werde. .. [D]er Staat ist verpflichtet, sie vor dieser realen Bedrohung zu schützen." ${ }^{6}$ Urgenda gewann im Dezember 2019 auch in letzter Instanz vor dem Hoge Raad. ${ }^{7}$

In Deutschland wurde die erste Klimaklage zunächst einmal vom Verwaltungsgericht Berlin abgewiesen. ${ }^{8}$ Jedoch auch das VG Berlin hat in seiner Entscheidung nicht jeden rechtlichen Ansatzpunkt abgelehnt, sondern durchaus betont, dass staatliche Schutzpflichten in diesem Kontext bestehen könnten. Diese seien prinzipiell auch verletzungsfähig, allerdings wäre dies nur dann anzunehmen, wenn staatliche Bemühungen von vornherein völlig aussichtslos und ungeeignet wären. Dies sei in Deutschland aber nicht der Fall.

Auch in anderen europäischen Ländern haben ähnliche Fälle vor nationalen Instanzen Gehör gefunden. So z.B. der Fall People v Arctic Oil in Norwegen, ${ }^{9}$ der Fall Klimaseniorinnen in der Schweiz, ${ }^{10}$ der Fall von Greenpeace in Österreich ${ }^{11}$ oder der Fall Klimaatzaak in Belgien. ${ }^{12}$ In Portugal planen von schweren Waldbränden betroffene Kinder, mehrere Staaten vor dem EGMR zu verklagen. ${ }^{13}$

6) The Hague Court of Appeal, Urt. v. 9.10.2018 - C/09/456689 13-1396 - Urgenda, s.: Stand 22.3.2020, abrufbar unter https:// elaw.org/nl.urgenda.15, \45. Der Gerichtshof erklärte, dass „,der Staat nach Art. 2 EMRK eine positive Verpflichtung zum Schutz des Lebens der Bürger in seinem Zuständigkeitsbereich hat, während Art. 8 EMRK die Verpflichtung zum Schutz des Rechts auf Wohnung und Privatleben begründet“. Der Gerichtshof ,ist der Ansicht, dass es angebracht ist, von einer realen Bedrohung des gefährlichen Klimawandels $z u$ sprechen, was zu dem ernsthaften Risiko führt, dass die gegenwärtige Generation von Bürgern mit Todesfällen und/oder einer Störung des Familienlebens konfrontiert wird. Wie der Gerichtshof bereits ausgeführt hat, ergibt sich aus den Art. 2 und 8 EMRK, dass der Staat verpflichtet ist, sie vor dieser realen Bedrohung zu schützen". Der Gerichtshof ist hier dem Verständnis des EGMR gefolgt, der Art. 2 im Umweltkontext in Ausnahmefällen für anwendbar erklärt, s.z.B.: EGMR, Urt. v. 17. 11. 2015 - Nr. 54000/11, 14350/05, 15245/05 und 16051/05 - Özel and Others v Turkey.

7) Hoge Raad, Urt. v. 20.12.2020 - 19/00135 - Urgenda, Stand 22.3.2020, abrufbar unter https://uitspraken.rechtspraak.nl/inz iendocument?id=ECLI:NL:HR:2019:2006.

8) VG Berlin, Urt. v. 31.10.2019 - $10 \mathrm{~K}$ 412.18, EnWZ 2020, 88.

9) In diesem Fall machten Greenpeace und eine Bürgerbewegung geltend, dass die Ausweitung der Ölförderung durch die Erteilung weiterer Genehmigungen in der Arktis gegen das durch die Verfassung geschützte Recht auf eine gesunde Umwelt und gegen die internationalen Verpflichtungen im Zusammenhang mit dem Klimawandel verstoße. Nach einem Urteil des Amtsgerichts Oslo ist Greenpeace in Berufung gegangen, Stand 22.3.2020, abrufbar unter http://www.climatechangenews.com/2018/02/ 05/greenpeace-appeal-norway-arctic-oil-drilling-case/.

10) In dieser Beschwerde vom 26.5.2017 argumentieren mehrere hundert Frauen im Pensionsalter, mit Unterstützung von Greenpeace Schweiz, dass das Versäumnis der Schweizer Regierung, die $\mathrm{CO}_{2}$-Emissionen wirksam zu reduzieren, einen Verstoß gegen Art. 2 und 8 EMRK darstelle. Sie argumentieren, dass ältere Menschen besonders anfällig für Hitzewellen seien, die durch den Klimawandel verursacht werden. Nachdem das Bundesverwaltungsgericht im Dezember 2018 gegen die Antragsteller entschieden hat, wurde am 21.1.2019 Berufung beim Bundesgericht eingelegt, Stand 17.3.2020, abrufbar unter https://klimaseniorinnen.ch.
Die Frage, ob derartige Fälle vor dem EGMR Erfolg haben können, hängt insbesondere davon ab, wie sich die bereits vom EGMR im Umweltbereich angewendeten positiven Schutzpflichten auf den Klimabereich übertragen lassen. Dass hier bereits ein Umdenken bei den Gerichten erfolgt, zeigt jüngst der Fall über die Expansion des Flughafens Heathrow. ${ }^{14}$

Ein Ziel dieses Artikels ist es aufzuzeigen, inwieweit über die Doktrin der positiven Schutzpflichten im Rahmen der EMRK eine Verantwortlichkeit für einzelne Staaten im Klimabereich zu begründen, obwohl das Phänomen des Klimawandels globaler Natur ist.

Dieser Artikel analysiert auf Grundlage der umweltrechtlichen Rechtsprechung des EGMR ${ }^{15}$ ob und inwieweit der EGMR für Klagen mit Klimabezug eine geeignete Entscheidungsinstanz sein könnte. Unter der aktuellen Rechtsprechung des EGMR bilden Fälle zum Umweltschutz und vor allem die Flughafenfälle ${ }^{16}$ eine Grundlage für die Diskussion, wie Staaten im Klimabereich zur Verantwortung gezogen werden können.

\section{Umweltschutz als Ausgangspunkt für klimabezogenen Fälle vor dem EGMR}

Auch wenn die EMRK kein ausdrückliches Recht auf eine saubere Umwelt enthält, ${ }^{17}$ werden einige EMRK-Garantien zunehmend dahingehend interpretiert: Spätestens seit dem Fall López Ostra, ${ }^{18}$ werden Art. 8 EMRK (Recht auf Achtung des Privat- und Familienlebens), zunehmend aber auch Art. 2 EMRK (Recht auf Leben), Art. 3 EMRK (Verbot von Folter und unmenschlicher Behandlung), Art. 6 EMRK (Recht auf ein faires Verfahren), Art. 10 EMRK

11) Klimaklage in Österreich, Stand 27.2.2020, abrufbar unter https://www.klimaklage.at/.

12) Vergleichbar mit dem Fall Urgenda ist in Belgien derzeit ebenso ein Fall anhängig, Stand 22.3.2020, abrufbar unter https://climate-laws.org/cclow/geographies/17/litigation_cases/.

13) Children -v- Goverments of Europe \& Climate Change, Stand 22.3.2020, abrufbar unter https://www.crowdjustice.com/case/ climate-change-echr/; In Europa und überall auf der Welt gibt es inzwischen weitere Klimaklagen, eine Übersicht dazu gibt es hier: Stand 22.3.2020, abrufbar unter https://climate-laws.org.

14) In einem Urteil, das dem Kampf gegen den Klimawandel oberste Priorität einräumt, hat das englische Berufungsgericht die geplante Expansion des Londoner Flughafens im Februar 2020 untersagt, Stand 22.3.2020, abrufbar unter https://www.judiciary. uk/wp-content/uploads/2020/02/Heathrow-judgment-on-planning-issues-27-February-2020.pdf. Somit steht das Urteil im Kontrast zu der Hatton-Rechtsprechung des EGMR, in der der Strasbourger Gerichtshof 2003 entschied, dass gesamtwirtschaftliche Interessen gegenüber den betroffenen Individualgütern, und in diesem Fall auch gegenüber umweltrechtlichen Erwägungen, höher zu bewerten seien.

15) In den meisten neueren Fällen liegen Interessenskonflikte zwischen dem Recht auf Eigentum und Umweltschutz vor, s.z.B. EGMR, Zulässigkeitsentscheidung v. 6.2.2018 - Nr. 23225/05 - Calancea and Others $v$ the Republic of Moldova; EGMR, Urt. v. 6.2.2018 - Nr. 36184/13 - Kristiana Ltd. v Lithuania; EGMR, Urt. v. 7.6.2018 - Nr. 44460/16 - O'Sullivan McCarthy Mussel Development Ltd v Ireland; EGMR, Urt. v. 12.6.2018 - Nr. 70520/10, 21920/10 and 41876/11 - Beinarovič and Others v Lithuania; EGMR, Urt. v. 6.9.2018 - Nr. 3401/09 - Dimitar Yordanov v Bulgaria; EGMR, Zulässigkeitsentscheidung v. 2. 4. 2019 - Nr. 54494/11, 67699/11 und 21251/12 - Pop and Others v Romania; EGMR, Zulässigkeitsentscheidung v. 26.11. 2019 - Nr. 64863/13 - Yaşar v Romania.

16) EGMR, Urt. v. 8.7.2003 - Nr. 36022/97 - Hatton and Others v the United Kingdom, $\$ 28$.

17) S. EGMR, Urt. v. 22.5. 2003 - Nr. 41666/98 - Kyrtatos v Greece, $\$ 52$; jedoch auch EGMR, Urt. v. 21.7.2009 - Nr. 12605/03 Leon and Agnieszka Kania v Poland, $\$ 98$.

18) EGMR, Urt. v. 9.12.1994 - Nr. 16798/90 - López Ostra v Spain. 
(Recht auf freie Meinungsäußerung) und Art. 13 EMRK (Recht auf wirksame Beschwerde) sowie Art. 1 des Protokolls Nr. 1 EMRK (Recht auf Eigentum) dahingehend ausgelegt, dass sie die Umwelt zumindest teilweise schützen. ${ }^{19}$

Der EGMR befasste sich bislang mit einer Vielzahl von Umweltproblemen, z.B. mit Umweltrisiken und Zugang $\mathrm{zu}$ Informationen, ${ }^{20}$ industrielle Umweltverschmutzung, ${ }^{21}$ Mobilfunkantennen, ${ }^{22}$ Flug-, ${ }^{23}$ Nachbarschafts- ${ }^{24}$ und StraBenverkehrslärm, ${ }^{25}$ Windkraftanlagen, ${ }^{26}$ Staudämme ${ }^{27}$ industrielle Lärmbelästigung, ${ }^{28}$ Schienenverkehr, ${ }^{29}$ Staubemissionen von Dieselfahrzeugen, ${ }^{30}$ Stadtentwicklung, ${ }^{31}$ Abfallentsorgung, ${ }^{32}$ Wasserversorgung, ${ }^{33}$ gesundheitsschädlichen Emissionen, ${ }^{34}$ Hochspannungsleitungen, ${ }^{35} \mathrm{Na}-$ turkatastrophen ${ }^{36}$ sowie dem Engagement von Umweltaktivisten. ${ }^{37}$ Ungeachtet dessen wurde in der Vergangenheit die Eignung der EMRK-Mechanismen zur Förderung des Umweltschutzes auch in Frage gestellt. ${ }^{38}$

\section{Die Relevanz der Doktrin der Positiven Pflichten}

\subsection{Die Rolle der positiven Verpflichtungen im Umweltbereich}

Die derzeitige Basis für die „Ökologisierung“ der EMRK ist neben der evolutionär-dynamischen Interpretation der EMRK ${ }^{39}$ die Doktrin der positiven Schutzpflichten. ${ }^{40} \mathrm{Ge}-$ mäß dieser Doktrin müssen die Staaten die Menschenrechte in ihrem Zuständigkeitsbereich aktiv schützen. Dies kann die Ergreifung vorbeugender und repressiver Maßnahmen gegen Menschenrechtsverletzungen einschließen (z.B. die Verabschiedung von Gesetzen) und zwar auch gegen solche Verletzungen, die nicht vom Staat selbst, sondern auch von Privatpersonen oder Unternehmen begangen werden. Im

19) Für einen Überblick über die umweltrechtliche Rechtsprechung des EGMR, s. Braig, Umweltschutz durch die Europäische Menschenrechtskonvention, 2013.

20) EGMR, Urt. v. 24.7.2014 - Nr. 13867/88 - Brincat and Others v Malta.

21) EGMR, Urt. v. 3. 5. 2011 - Nr. 6854/07 - Apanasewicz v Poland.

22) EGMR, Zulässigkeitsentscheidung v. 17.1.2006 - Nr. 42756/02 - Luginbühl v Switzerland.

23) EGMR, Urt. v. 13.12. 2012 - Nr. 3675/04 und 23264/04 - Flamenbaum and Others $v$ France.

24) EGMR, Urt. v. 22.11.2011 - Nr. 24202/10 - Zammit Maempel and Others v Malta; EGMR, Urt. v. 161.2018 - Nr. 23383/12 Cuenca Zarzoso v Spain.

25) EGMR, Urt. v. 21.7.2011 - Nr. 38182/03 - Grimkovskaya v Ukraine.

26) EGMR, Zulässigkeitsentscheidung v. 26.2.2008 - Nr. 37664/04 - Fägerskiöld v Sweden; anhängige Beschwerde: EGMR Nr. 52499/11 - Vecbaštika and Others v Latvia.

27) EGMR, Zulässigkeitsentscheidung v. 29.1.2019 - Nr. 6080/06 Ahunbay and Others v Turkey.

28) EGMR, Urt. v. 1.7.2008 - Nr. 71146/01 - Borysiewicz v Poland

29) EGMR, Urt. v. 18.6.2013 - Nr. 50474/08 - Bor v Hungary.

30) EGMR, Zulässigkeitsentscheidung v. 12.5.2009 - Nr. 18215/06 - Greenpeace e. V. and Others v Germany.

31) EGMR, Urt. v. 22.5.2003 - Nr. 41666/98 - Kyrtatos v Greece.

32) EGMR, Urt. v. 7.4.2009 - Nr. 6586/03 - Brânduse v Romania; anhängige Beschwerden: EGMR - Nr. 35648/10 - Locascia and Others v Italy und EGMR - Nr. 39742/14 - Di Caprio and Others v Italy.

33) EGMR, Urt. v. 4. 9.2014 - Nr. 42488/02 - Dzemyuk v Ukraine.

34) EGMR, Urt. v. 24.1.2019 - Nr. 54414/13 - Cordella and Others v Italy.

35) Anhängige Beschwerde: EGMR - Nr. 41892/19 und 41893/19 Thibaut v France.

36) EGMR, Zulässigkeitsentscheidung v. 28.11.2006 - Nr. 76973/01 - Murillo Saldias and Others v Spain; EGMR, Zulässigkeitsentscheidung v. 24.3.2015 - Nr. 9713/13 - Viviani and Others v Italy.

37) Anhängige Beschwerden: EGMR - Nr. 22515/14 - Bryan and Others v Russia; EGMR - Nr. 56176/18 - Burestop 55v France; EGMR - Nr. 56189/19-Asodera v France; EGMR - Nr. 56232/18 - Mirabel-Lne v France; EGMR - Nr. 56236/18 - Fédération
Folgenden skizzieren wir die positiven Schutzpflichten, mit denen der EGMR zur Gestaltung des Umweltrechts in Europa beigetragen hat $\mathrm{t}^{41}$ und zeigen auf, inwieweit diese sich auf den Klimabereich übertragen lassen können.

Gemäß der EMRK kommt den Staaten eine positive Verpflichtung zu, fehlende und ineffiziente staatliche (Umweltschutz-)Maßnahmen angemessen zu adressieren. ${ }^{42}$

\subsection{Die im Umweltbereich angewendete Doktrin der positiven Schutzpflichten als Grundlage für Beschwerden im Klimabereich?}

Die meisten positiven Schutzplichten und Verfahrensgaran$\operatorname{tien}^{43}$ die der EGMR in den letzten Jahren im Umweltschutzbereich etabliert hat (oftmals unter Art. 8 EMRK), können sich wie folgt auf den Klimabereich übertragen lassen:

\subsubsection{Gewähr eines Informationszugangs}

über die Risiken des Klimawandels

Nach der derzeitigen Rechtsprechung des EGMR hat die Öffentlichkeit ein Recht auf Information über Umweltrisiken, die möglicherweise erhebliche Gesundheitsschäden verursachen können. ${ }^{44}$ Die Staaten sind verpflichtet, relevante Umweltinformationen zu sammeln und ein wirksames Informationssystem für die interessierte Öffentlichkeit zu gewährleisten. ${ }^{45}$ Gemäß den Ausführungen des EGMR muss die Öffentlichkeit, bei einer unabhängigen Stelle, einen effektiven Zugang zu Ergebnissen von Studien (z. B. UVPs) und Informationen haben, die eine Bewertung des Risikos ermöglichen, dem sie ausgesetzt ist. ${ }^{46}$

Es kann argumentiert werden, dass dies auch Risiken einschließt, die durch den Klimawandel entstehen. Der Zugang zu Informationen über allgemeine und spezifische

Réseau Sortir du Nucléaire v France; EGMR - Nr. 56241/18 - Les Habitants vigilants du canton de Gondrecourt v France; EGMR - Nr. 56247/18 - CEDRA 52v France.

38) Theil, NuR 2014, 330 .

39) D.h. ein gesellschaftlicher Wandel, z.B. hin zu einem klimabewussteren Handeln, kann sich auch in der Rechtsprechung wiederspiegeln, vgl. Piela, ,60 Jahre Europäische Menschenrechtskonvention: Die Konvention als ,,living instrument"“, Zeitschrift für Menschenrechte, 2015, S. 190.

40) In der Regel umfassen positive Schutzpflichten Verpflichtungen, die der Staat erfüllen muss, um ,die Schritte festzulegen, die zu unternehmen sind, um die Einhaltung der Konvention unter gebührender Berücksichtigung der Bedürfnisse und Ressourcen der Gemeinschaft und des Einzelnen sicherzustellen" (EGMR, Urt. v. 18.3.2011 - Nr. 30814/06 - Lautsi v Italy), S61; positive Verpflichtungen bilden den Gegensatz zu negativen Verpflichtungen, bei denen der Staat hauptsächlich verpflichtet ist, nicht in die persönliche Sphäre eines Individuums einzugreifen; s. Mowbray, ,The Creativity of the European Court of Human Rights', Human Rights Law Review, 2005, 57.

41) Cf. Antypas et al., ,Linking Environmental Protection, Health, and Human Rights in the European Union, An Argument in Favour of Environmental Justice Policy،, Environmental Law \& Management, 2008,S. 8; s. auch EGMR, Urt. v. 24.1.2019Nr. 54414/13 - Cordella and Others v Italy, sowie den anhängigen Fall: EGMR - Nr. 35648/10 - Locascia and Others v Italy.

42) EGMR, Urt. v. 20.5.2010 - Nr. 61260/08-Oluic v Croatia, \66.

43) Für den Unterschied zwischen prozeduralen und substantiellen Schutzpflichten im Kontext der positiven Verpflichtungen, s. Akandji-Kombe, Positive obligations under the European Convention on Human Rights, A guide to the implementation of the European Convention on Human Rights, Council of Europe 2007.

44) EGMR, GK, Urt. v. 19.2.1998 - Nr. 14967/89 - Guerra and Others v Italy, \60; EGMR, Urt. v. 9.6.1998 - Nr. 23413/94 L. C. B. v United Kingdom.

45) EGMR, Urt. v. 9.6.1998 - Nr. 21825/93, 23414/94 - McGinley and Egan v United Kingdom, $\$ 99,102$

46) EGMR, Urt. v. 7.4.2009 - Nr. 6586/03 - Brânduse v Romania, \74; EGMR, 19. 10.2005 - Nr. 32555/96 - Roche v United Kingdom, \$169; EGMR, Urt. v. 27.1.2009 - Nr. 67021/01 - Tătar v Romania, \$115-124. 
Aspekte des Klimawandels ist für den Einzelnen möglicherweise von wesentlicher Bedeutung, um vorbeugende Maßnahmen zu ergreifen. Ein solcher Zugang zu angemessenen und spezifischen Informationen könnte es z.B. Bewohnern oder Unternehmern in Küstennähe ermöglichen, bestimmte, von Klimarisiken (z. B. Überschwemmungen) bedrohte Wohnorte oder Geschäftsstätten für den Kauf von Immobilien zu vermeiden (so kann eine Wertminderung des Eigentums, geschützt durch Art. 1 des ersten Zusatzprotokolls verhindert werden) oder gefährdete Gebiete rechtzeitig zu verlassen (umso möglicherweise einer lebensbedrohlichen Katastrophe zu entkommen, die analog zur Rechtsprechung in den Fällen Öneryildiz ${ }^{47}$ oder Kolyadenko $o^{48}$ z. B. unter Art. 2 oder Art. 8 fallen könnte).

\subsubsection{Beteiligung der Öffentlichkeit an}

Entscheidungsprozessen im Klimabereich

Nach ständiger Rechtsprechung des EGMR müssen Staaten die Öffentlichkeit an Entscheidungsprozessen, die erhebliche Umweltauswirkungen haben können (z. B. gröBere Infrastrukturprojekte), durch bestimmte Verfahren beteiligen. ${ }^{49}$

Somit scheint es auch möglich, dass der EGMR eine Öffentlichkeitsbeteiligung fordern könnte, z.B. wenn es um Küstenzonenmanagement oder andere Klimaanpassungsmaßnahmen geht. So könnte der EGMR argumentieren, dass Anpassungsmaßnahmen häufig orts- und kontextspezifisch sind, Auswirkungen auf eine relativ begrenzte Interessengruppe haben und Know-How erfordern, das auf die örtlichen Gegebenheiten zugeschnitten ist.

3.3.3 Zugang zu Gerichten in Bezug

auf Fragen des Klimawandels.

Insbesondere gemäß Art. 6 EMRK (sowie auch gemäß Art. $8 \mathrm{EMRK}^{50}$ ) muss ein umfassender Zugang zu Gerichten in Umweltangelegenheiten gewährt werden, der insbesondere folgende Elemente umfasst: ${ }^{51}$ Erstens muss den Betroffenen die Möglichkeit eingeräumt werden rechtliche Schritte einzuleiten, wenn staatliche Umweltschutzmaßnahmen als für nicht ausreichend erachtet werden oder ganz unterlassen wurden ${ }^{52}$ bzw. erachtet wird, dass diese im Planungsprozess nicht ausreichend berücksichtigt wurden. ${ }^{53}$ Zweitens muss gewährleistet sein, dass die Frage der staatlichen Verantwortung für gerügte Umweltschäden vor einem Gericht oder einer Verwaltungsbehörde wirksam untersucht werden kann. ${ }^{54}$ Drittens darf ein Gerichtsverfahren nicht länger als nötig hinausgezögert werden. ${ }^{55}$ Viertens müssen gerichtliche Entscheidungen, die umweltschädigende Tätigkeiten als rechtswidrig einstufen, auch umgesetzt werden. ${ }^{56}$

Bezogen auf den Klimawandel könnte der umfassende Zugang zur Gerichten z. B. vorsehen, dass vor der Durchführung von Industrietätigkeiten, die erheblich zum Klimawandel beitragen (z.B. durch die Freisetzung großer Emissionsmengen), die Öffentlichkeit die Möglichkeit haben muss, ein Gerichtsverfahren einzuleiten, wenn sie feststellt, dass ihre Interessen im Planungsprozess nicht angemessen berücksichtigt worden sind.

\subsubsection{Pflicht zur Verabschiedung}

einer Klimaschutzgesetzgebung

Nach der ständigen Rechtsprechung des EGMR müssen Staaten Umweltgesetze erlassen, um gefährliche Tätigkeiten angemessen zu reglementieren und um Entscheidungsprozesse auf Grundlage von Studien und Untersuchungen zu gewährleisten, die die Bewertung von möglicherweise bestehenden Umwelt- und Gesundheitsrisiken erlauben. ${ }^{57}$

Bezogen auf den Klimakontext scheint es wahrscheinlich, dass der EGMR im Lichte dieser Rechtsprechung zu dem Schluss kommen könnte, dass die positive Verpflichtung zur Verabschiedung von Umweltschutzgesetzen für die Staaten auch beinhaltet, eine angemessene Klima- gesetzgebung zu verabschieden - zumal davon ausgegangen wird, dass der Klimawandel auch erhebliche negative Gesundheitsrisiken bzw. gar unmittelbare Gesundheitsgefahren mit sich bringt. ${ }^{58}$

Es wird sich möglicherweise die Frage stellen lassen, ob das derzeit geplante Klimaschutzpaket der Bundesregierung im Lichte der EMKR bereits als angemessen genug gilt, obwohl z.B. (bislang nicht veröffentlichte) Gutachten nahelegen, dass die Bundesregierung damit ihre Ziele für 2030 nicht erreichen kann. ${ }^{59}$ Auch könnte die Frage aufgeworfen werden, ob, vor dem Hintergrund der Tatsache, dass viele Staaten ihren im Jahr 2015 im Pariser Übereinkommen festgehaltenen Verpflichtungen nicht nachzukommen scheinen, ein für eine EMRK-Konformität nötiger ausreichend fairer Ausgleich zwischen widerstreitenden Interessen geschaffen wurde. ${ }^{60}$ Denn gemäß der Rechtsprechung des EGMR müssen staatliche Schutzmaßnahmen in einem absehbaren Zeitraum wirksam werden. ${ }^{61}$

\subsection{5 Überwachungs- und Kontrollpflichten}

im Klimaschutzbereich

Da in der Regel nur dann, wenn Staaten über funktionierende Kontrollsysteme von umweltschädlichen Gefahren auf verschiedenen Ebenen verfügen, auch tatsächlich eine angemessene Warnung der Öffentlichkeit vor bevorstehenden Gefahren erfolgen kann, kennt der EGMR auch eine Pflicht zur Kontrolle und Überwachung eines möglicherweise mangelhaften Vollzugs des bestehenden Umweltrechts. ${ }^{62}$

In Kontext des Klimawandels könnte dies bedeuten, dass eine Verletzung der EMRK vorliegt, wenn gravierende

47) EGMR, Urt. v. 30.11.2004 - Nr. 48939/99 - Öneryildiz v Turkey, $\$ 89-90,100-102$.

48) EGMR, Urt. v. 28.2.2012 - Nr. 17423/05, 20534/05, 20678/05, 23263/05, 24283/05 und 35673/05 - Kolyadenko and Others v Russia.

49) EGMR, Urt. v. 2.11.2006 - Nr. 59909/00 - Giacomelli v Italy, §82; EGMR, GK, Urt. v. 8.7.2003-Nr. 36022/97 - Hatton and Others $\vee$ United Kingdom.

50) Z. B: EGMR, Urt. v. 21.7.2011 - Nr. 38182/03 - Grimkovskaya v Ukraine, $\$ 69$.

51) S.: EGMR, Urt. v. 11.3.2014 - Nr. 52067/10 und 41072/11 - Howald Moor and Others v Switzerland; EGMR, Urt. v. 25.9. 2014 - Nr. 29878/09 - Karin Andersson and Others v Sweden; anhängige Beschwerde EGMR - Nr. 19732/17 - Stichting Landgoed Steenbergen and Others $\mathrm{v}$ the Netherlands.

52) Hier auch Bezug nehmend auf Art. 8 EMRK, EGMR, Urt. v. 10.11.2004 - Nr. 46117/99 - Taşkın v Turkey, $\$ 119$, \$130.

53) EGMR, Urt. v. 27.1.2009 - Nr. 67021/01 - Tătar v Romania, $\$ 124$

54) EGMR, Urt. v. 20.3.2008 - Nr. 15339/02, 21166/02, 20058/02, $11673 / 02$ and $15343 / 02$ - Budayeva and Others v Russia, $\$ 160 \mathrm{ff}$

55) EGMR, Urt. v. 25.11.2010 - Nr. 43449/02, 21475/04 - Mileva and Others v Bulgaria, $\$ 100$.

56) EGMR, Urt. v. 2.11.2006 - Nr. 59909/00 - Giacomelli v Italy, \$94; EGMR, Urt. v. 5.6.2007 - Nr. 17381/02 - Lemke v Turkey, \52; EGMR, Urt. v. 19.6.2018 - Nr. 25680/05 - Bursa Barosu Başkanlığ 1 and Others v Turkey.

57) EGMR, Urt. v. 27.1.2009 - Nr. 67021/01 - Tătar v Romania, $\$ 88$.

58) So geht z.B. eine jüngste Studie davon aus, dass ein weltweiter Kohleausstieg 1,4 Millionen Todesfälle verhindern würde, s. Rauner/Bauer/Dirnaichner et al., ,Coal-exit health and environmental damage reductions outweigh economic impacts', 2020, Nature Climate Change https://doi.org/10.1038/s41558-020-0728-x.

59) Diese Gutachten liegen dem Spiegel vor, Stand 5.3.2020, abrufbar unter https://www.spiegel.de/wirtschaft/soziales/andreasscheuer-raeumt-nach-kritischen-gutachten-nachholbedarf-beiklimaschutz-ein-a-50b0a27b-bda3-40fc-8226-4f6f699ea3c2

60) S. EGMR, Urt. v. 9.6.2005 - Nr. 55723/00 - mutatis mutandis, Fadeyeva v Russia, $\$ 132 \mathrm{ff}$.

61) EGMR, Urt. v. 26. 10.2006 - Nr. 53157/99, 53247/99, 53695/00 und $56850 / 00-$ Ledyayeva and Others v Russia, $\$ 110$.

62) EGMR, Urt. v. 7. 4. 2009 - Nr. 6586/03 - Brânduşe v Romania, $\$ 71$. 
Defizite beim Vollzug klimarelevanter Gesetze, z.B. bei Treibhausgas-Emissionshandelsgesetzen zu Tage treten. Im Fall Kolyadenko kam der EGMR bereits zu dem Schluss, dass dies der Fall ist, wenn ein Staat kein ausreichendes Hochwasserrisikomonitoring betreibt. ${ }^{63}$ Die Argumentation des Gerichtshofs könnte auch auf Szenarien zu einem durch den Klimawandel verursachten Meeresspiegelanstieg angewendet werden, sofern der Staat den Meeresspiegelanstieg nicht wirksam überwacht und letzterer eine erhebliche Gefahr für das Leben und das Eigentum der Küstenbewohner und dort ansässiger Unternehmen mit sich bringt.

\subsubsection{Pflicht zur Risikobewertung und -evaluierung} im Lichte des Vorsorgeprinzips

Bei komplexen Umweltproblemen muss dem EGMR zufolge, ,der Entscheidungsprozess angemessene Untersuchungen und Studien umfassen, um [...] die Auswirkungen von Aktivitäten, die sich möglicherweise schädigend auf die Umwelt auswirken und Individualrechte verletzen können, voraussagen zu können [...].“64

Bei Bestehen eines erheblichen Risikos für die menschliche Gesundheit und das Wohlergehen besteht eine positive staatliche Schutzpflicht zur Risikobewertung und -evaluierung, ${ }^{65}$ z. B. in Form von Umweltverträglichkeitsprüfungen (die dem EGMR zufolge auch relativ detaillierten Anforderungen genügen müssen). ${ }^{66}$ Diese Schutzpflicht wird im Fall Tătar, insbesondere mit Verweis auf das Vorsorgeprinzip begründet. ${ }^{67}$

Die Vermutung liegt nahe, dass der EGMR beim Vorliegen schwerwiegender und wesentlicher Klimarisiken mit Auswirkungen auf Gesundheit und Wohlergehen von Beschwerdeführern die hier ausgeführten Risikobewertungsund -evaluierungspflichten ebenfalls zur Anwendung kommen lassen würde. Das Vorsorgeprinzip könnte somit - in der Form wie es derzeitig vom EGMR interpretiert wird - wichtige Leitlinien für den Umgang mit wissenschaftlichen Unsicherheiten hinsichtlich der langfristigen Auswirkungen des Klimawandels liefern.

\subsubsection{Pflicht zum Treffen angemessener}

Sicherheitsvorkehrungen im Klimabereich

Aus der Rechtsprechung des EGMR lässt sich eine Verpflichtung für die Staaten ableiten, angemessene Sicherheitsvorkehrungen (z.B. in Form der Installierung von Warnsystemen, der Instandhaltung von bestehender Infrastruktur ${ }^{68}$ oder Notfallschutzmaßnahmen nach Umweltkatastrophen $^{69}$ ) für potenziell gefährliche Situationen für die Umwelt und in fine für den Menschen zu treffen. ${ }^{70}$ Dies gilt dem EGMR zufolge auch für Umweltrisiken, die keinen direkten Einfluss auf die Gesundheit und das Wohlbefinden der Menschen haben. ${ }^{71}$

Ähnlich wie der EGMR im Fall Öneryildiz (in der der Gerichtshof eine Verletzung von Art. 2 EMRK festgestellt hat $)^{72}$ argumentierten übrigens die Richter im Fall Urgenda: Sobald durch den Klimawandel ein hohes Risiko und damit einhergehend schwerwiegende und lebensbedrohliche Folgen für Mensch und Umwelt bestehen, sei der Staat verpflichtet, seine Bürger durch geeignete und wirksame Maßnahmen zu schützen. ${ }^{73}$

Wenn also vorhersehbare Gefahren durch den Klimawandel bestehen, würde der EGMR möglicherweise dieselben Kriterien anwenden wie in seiner umweltrechtlichen Rechtsprechung und Staaten verpflichten, angemessene Sicherheitsvorkehrungen $\mathrm{zu}$ treffen (z.B. das Bauen von Schutzwallen).

Auch hier wird sich jedoch die Frage stellen, was gemäß dem EGMR als angemessen gelten würde. Der Bau eines 161 Kilometer langen Damms, der den Ärmelkanal zwischen Bretagne und Cornwall schließt, sowie einer zweiten, fast 500 Kilometer langen Barriere in der nördlichen Nordsee zwischen Schottland und Norwegen, wie er jüngst von Forschern angeregt wurde? ${ }^{74}$ Das Ministerium für Um- welt, Klima und Energiewirtschaft in Baden-Württemberg hat umfangreiche Vulnerabilitätsanalysen verschiedener vom Klimawandel betroffener Bereiche durchgeführt und verschiedene Schutzmaßnahmen vorgeschlagen. ${ }^{75}$ Müssen diese Maßnahmen, zumindest in Teilen, umgesetzt werden um künftig den Anforderungen der EMRK zu genügen?

3.3.8 Verfolgung und Bestrafung von klimaschädlichem Verhalten

Im Falle einer Umweltbeeinträchtigung, die a priori nicht im Einklang mit der EMRK steht, muss der Staat dem EGMR zufolge auch wirksame Ermittlungen einleiten, ${ }^{76}$ um die für illegale Umweltauswirkungen Verantwortlichen auf allen Ebenen zu identifizieren, ${ }^{77}$ gegebenenfalls vor Gericht zu bringen und angemessen zu bestrafen. ${ }^{78} \mathrm{Die}$ verhängten Sanktionen müssen geeignet sein, die Verursacher zu veranlassen, erforderliche Umweltschutzmaßnahmen zu ergreifen. ${ }^{79}$

Da ein schwerwiegender Verstoß gegen die Gesetze zur Eindämmung des Klimawandels auch negative Auswirkungen auf die Umwelt haben kann, ist es wahrscheinlich, dass der EGMR vergleichbare Verpflichtungen in Bezug auf die Verfolgung und Bestrafung von Akteuren, die das Klima schädigen, etablieren würde. In manchen Mitgliedsstaaten könnte die Frage aufkommen, inwieweit die verhältnismäßig milden Strafen und Bußgelder für Umweltdelikte im Klimabereich als angemessen im Sinne der EMRK angesehen werden können. Die Tatsache, dass in Deutsch-

63) EGMR, Urt. v. 28. 2.2012 - Nr. 17423/05, 20534/05, 20678/05, 23263/05, 24283/05 und 35673/05 - Kolyadenko and Others v Russia.

64) EGMR, Urt. v. 28.3.2006 - Nr. 46771/99 - Öçkan and Others v Turkey, \43; dazu auch EGMR, Urt. v. 2.11.2006 Nr. 59909/00 - Giacomelli v Italy, \96.

65) EGMR, Urt. v. 27.1.2009 - Nr.67021/01 - Tătar v Romania, S114; s. auch EGMR, Zulässigkeitsentscheidung v. 3.7.2007 Nr. 32015/02 - Gaida v Germany.

66) EGMR, Urt. v. 27.1.2009 - Nr. 67021/01 - Tătar v Romania, ऽ $114-115$.

67) EGMR, Urt. v. 27.1.2009 - Nr. 67021/01 - Tătar v Romania, $\$ 70$.

68) EGMR, Urt. v. 20.3. 2008 - Nr. 15339/02, 21166/02, 20058/02, 11673/02 und 15343/02 - Budayeva and Others v Russia, \$116; s. auch EGMR, Urt. v. 16. 11.2004 - Nr. 4143/02 - Moreno Gómez v Spain, $\$ 60$.

69) EGMR, Urt. v. 27.1.2009 - Nr. 67021/01 - Tătar v Romania, Beschwerde, $₫ 114$.

70) EGMR, Urt. v. 5. 12.2013 - Nr. 52806/09 und 22703/10 - Vilnes and Others v Norway; EGMR, Urt. v. 9.6.2005 - Nr. 55723/00 - Fadeyeva v Russia, $\$ 89$.

71) EGMR, Urt. v. 25.11.2010 - Nr. 43449/02, 21475/04 - Mileva and Others v Bulgaria, \$97, 99.

72) EGMR, GK, Urt. v. 30.11.2004 - Nr. 48939/99 - Öneryildiz v Turkey, §89-90, 100-102.

73) Rechtbank Den Haag, The District Court of the Hague, Urgenda C/09/456689, 13-1396, 24. 5.2015, \$4.74.

74) S. z.B. „Schutz vor Überflutung: Forscher entwerfen gigantischen Damm für die Nordsee", Stand 22.3.2020, abrufbar unter https://www.spiegel.de/wissenschaft/mensch/klimawandel-forscher-planen-gigantischen-damm-fuer-die-nordsee-a-74c7c8efe362-46df-91b0-a181dc1ee963.

75) S. Strategie zur Anpassung an den Klimawandel in Baden-Württemberg, Stand 22.3.2020, abrufbar unter https://um.badenwuerttemberg.de/fileadmin/redaktion/m-um/intern/Dateien/ Dokumente/4_Klima/Klimawandel/Anpassungsstrategie.pdf.

76) EGMR, Urt. v. 30.11.2004 - Nr. 48939/99 - Öneryildiz v Turkey.

77) EGMR, Urt. v. 20.3.2008 - Nr. 15339/02, 21166/02, 20058/02, 11673/02 und 15343/02 - Budayeva and Others v Russia, \132.

78) EGMR, Urt. v. 8. 1.2009- Nr. 12050/04 - Mangouras v Spain, $\$ 41$

79) EGMR, Urt. v. 9.6.2005 - Nr. 55723/00 - Fadeyeva v Russia, $\$ 130 ;$ s. auch die anhängige Beschwerde, EGMR - Nr. 35648/10 - Locascia and Others v Italy. 
land z.B. ein Defizit beim Vollzug des Umwelt(straf)rechts besteht ${ }^{80}$ könnte auf eine EMRK-Konformität untersucht werden. Dabei könnte man die Faktoren, die dieses befördern, z. B. die Kapazitätsverringerungen in Behörden, ${ }^{81}$ fehlende Spezialisierung von Behörden, der technisch-naturwissenschaftliche Charakter von Ermittlungen zu Umweltstraftaten, der komplexe Rechtsrahmen sowie die Tatsache, dass Umweltstraftaten im Vergleich zu anderen Typen von Kriminalität häufig keine hohe Priorität beim Vollzug haben, ${ }^{82}$ genauer unter die Lupe nehmen.

\section{Die möglichen Grenzen positiver Schutzpflichten im Bereich des Klimawandels}

Die Anwendung der positiven Schutzpflichten im Klimabereich ist jedoch auch in mancherlei Hinsicht begrenzt: Tatsächlich ist der anthropozentrisch ausgerichtete Umweltschutz, den der EGMR bietet, dadurch begrenzt, dass nur diejenigen Beschwerdeführer, die persönlich von einer negativen Umweltbeeinträchtigung beeinflusst sind, diese auch vor den EGMR bringen können. Darüber hinaus schützt das Straßburger Rechtssystem vor konkreten und drohenden Gefahren, wendet jedoch nicht in jedem Falle potenzielle Risiken ab.

Erstens muss die Bevölkerung nach der Rechtsprechung des EGMR für das Funktionieren der heutigen Gesellschaft ein gewisses Risiko eingehen (wenn umwelt- oder klimaschädigende Tätigkeiten z. B. im öffentlichen Interesse sind). ${ }^{83}$ Zweitens hat der EGMR in der Vergangenheit argumentiert, dass die Verhältnismäßigkeit im Umweltbereich - und

80) Status quo und Weiterentwicklung des Umweltstrafrechts und anderer Sanktionen: Instrumente zur Verbesserung der Befolgung von Umweltrecht (Compliance), Abschlussbericht, Stand 21.3.2020, abrufbar unter https://www.umweltbundesamt.de/ sites/default/files/medien/1410/publikationen/2019-11-07_ texte_135-2019_umweltstrafrecht_0.pdf.

81) Umweltordnungswidrigkeiten und Umweltstrafen, Stand 21.3.2020, abrufbar unter https://www.umweltbundesamt.de/ themen/nachhaltigkeit-strategien-internationales/umweltrecht/ umweltordnungswidrigkeiten-umweltstraftaten.

82) Status quo und Weiterentwicklung des Umweltstrafrechts und anderer Sanktionen: Instrumente zur Verbesserung der Befolgung von Umweltrecht (Compliance), Abschlussbericht, Stand 21.3.2020, abrufbar unter https://www.umweltbundesamt.de/ sites/default/files/medien/1410/publikationen/2019-11-07_ texte_135-2019_umweltstrafrecht_0.pdf.

83) EGMR, Urt. v. 21.7.2009 - Nr. 12605/03 - Kania v Poland, $\$ 100 \mathrm{ff}$; der EGMR hat bereits mehrfach betont, dass finanzielle Interessen gegenüber dem Umweltschutz nicht prioritär behandelt werden dürften, s.z.B. EGMR, Urt. v. 8.7.2009 Nr. 1411/03 - Turgut v Turkey, $\$ 90$.

84) EGMR, Urt. v. 30.3.2010 - Nr. 19234/04 - Băcilă v Romania, \$69; EGMR, GK, Urt. v. 2.10.2001 - Nr. 36022/97 - Hatton $\mathrm{v}$ the United Kingdom,- Opinion of Judge Greve; EGMR, Zulässigkeitsentscheidung v. 9.11.2004 - Nr. 31888/03 - Ward $\mathrm{v}$ the United Kingdom; EGMR, Zulässigkeitsentscheidung v. 9. 9.2014 - Nr. 55396/07 - Chis v Romania; die Frage, was für ein solches Funktionieren einer Industriegesellschaft angesichts von gravierenden Menschenrechtsverletzungen notwendig ist, wird jedoch möglicherweise vor dem Hintergrund der CoronaPandemie neu bewertet werden.

85) EGMR, Urt. v. 28.5.2002 - Nr. 29695/96 - Gronus v Poland; EGMR, 10.11.2004 - Nr. 46117/99 - Taškın v Turkey, \$113; cf. Thienel, ,The Burden and Standard of Proof in the European Court of Human Rights", 50 German Yearbook of International Law 2007, 2008, S. 543. Ein Eindruck davon, wie der EGMR mit dieser Herausforderung umgeht, findet sich in: EGMR, Urt. v. 27.1.2009 - Nr. 67021/01 - Tătar v. Rumänien, Partially Dissenting Opinion of Juge Zupančič joined by Judge Gyulumyan.

86) EGMR, Zulässigkeitsentscheidung v. 6.2.2018 - Nr. 23225/05 - Calancea and Others v the Republic of Moldova.

87) EGMR, Urt. v. 2.10.2001 - Nr. 36022/97 - Hatton and Oters v United Kingdom, \$127; EGMR, Zulässigkeitsentscheidung dies dürfte auch für Szenarien des Klimawandels der Fall sein - eine entscheidende Rolle spiele, da viele potenziell umweltschädliche Aktivitäten, einschließlich solcher, die für Dritte schädlich sind, zugelassen werden müssten um das Funktionieren einer Industriegesellschaft zu gewährleisten. ${ }^{84}$ Drittens können zukünftige Klimaklagen in Strasbourg an einer hohen Beweislast, unzureichenden Kausalzusammenhängen oder einer zu geringen Schadenseintrittswahrscheinlichkeit scheitern. ${ }^{85}$ Viertens hängt die Frage, ob ein Staat positive Maßnahmen ergreifen muss, von verschiedenen Umständen ab, wie z. B. der Intensität (Schwere und Dauer) der Beeinträchtigung ${ }^{86}$ - für Personen mit einer ,durchschnittlichen Empfindlichkeit" ${ }^{\text {87 }}$ - sowie ihren physischen und psychischen Auswirkungen; ${ }^{88}$ d. h. z. B. minimale Klimaerwärmungen, für sich isoliert gesehen, dürften - selbst wenn wissenschaftlich nachgewiesen - hier nicht ausreichend sein. Fünftens ist nach der Rechtsprechung des EGMR der Ermessensspielraum von Staaten in Umweltangelegenheiten besonders groß. ${ }^{89}$ Sechstens können potenzielle Opfer und NGOs mangels direkter Betroffenheit nur in begrenztem Umfang von den positiven Schutzpflichten der EMRK profitieren. ${ }^{90}$ Siebtens ist die derzeitige Doktrin der Extraterritorialität ${ }^{91}$ sehr eng gefasst und umfasst im Wesentlichen staatliche Akteure. ${ }^{92}$ Dies erschwert es potenziellen, aus Drittstaaten stammenden Opfern von Bedrohungen, die im Kontext des Klimawandels stehen (z.B. Klimamigranten), von diesem Schutz zu profitieren. ${ }^{93}$ Achtens: Da der Klimawandel sowohl von staatlichen als auch von privaten Akteuren auf der ganzen Welt verursacht wird, kann es schwierig sein, die Verantwortung eines einzelnen Staates

v. 3.7. 2007 - Nr. 32015/02 - Gaida v Germany, \$22; was dies z. ${ }^{\circ}$ B. für hitzeempfindliche Personen oder Personen mit einem überdurchschnittlichen Alter bedeutet, die besonders unter den Folgen des Klimawandels zu leiden haben, ist offen.

88) EGMR, Urt. v. 9.6.2005 - Nr. 55723/00 - Fadeyeva v Russia, ऽ68ff.

89) S. Müllerová, ,Environment Playing Short-handed: Margin of Appreciation in Environmental Jurisprudence of the European Court of Human Rights', Review of European Comparative \& International Environmental Law, 2015, 83.

90) EGMR, Zulässigkeitsentscheidung v. 28.3.2006 - Nr. 75218/01 - Collectif national d'information et d'opposition à l'usine Melox - Collectif stop Melox et Mox, \4; EGMR, Urt. v. 19.6.2018 - Nr. 25680/05 - Bursa Barosu Başkanlı̆̆ 1 and Others v Turkey; s. jedoch auch EGMR, Urt. v. 24.2.2009 - Nr. 49230/07 - L'Erablière A.S.B.L. v Belgium, und neuere Entwicklungen in anderen Kontexten, z.B., EGMR, Urt. v. 4.12.2015 Nr. 47143/06 - Roman Zakharov v Russia, in diesem Fall hat der EGMR festgehalten, dass der Beschwerdeführer die Opfereigenschaft im Sinne der EMRK erfülle, obwohl er nicht selbst Opfer von den von ihm beklagten Überwachungsmaßnahmen geworden war.

91) EGMR, Urt. v. 7.7.1989 - Nr. 23413/94 - Soering v United Kingdom; Heiskanen/Viljanen, ,Reforming the Strasbourg Doctrine on Extraterritorial Jurisdiction in the Context of Environmental Protection', European Law Reporter, 2014, 285; Besson, ,The Extra-territoriality of the European Convention on Human Rights. Why Human Rights Depend on Jurisdiction and What Jurisdiction Amounts to', 25:4 Leiden Journal of International Law, 2012, 857-84.

92) In Analogie zu dem Fall, EGMR, Urt. v. 8. 7.2004-Nr. 48787/99 - Ilașcu v Moldova, ist es jedoch denkbar, dass im Klimabereich künftig auch Konzerne, die de facto die Kontrolle über bestimmte Gegenden haben, als staatliche Akteure angesehen werden.

93) Mit Bezug auf Abschiebungen in durch die negativen Auswirkungen des Klimawandels unbewohnbar gemachte Regionen, die sich außerhalb der Konventionsstaaten befinden, ist jedoch möglicherweise der Fall EGMR, Urt. v. 2.5.1997 - Nr. 30240/96 D. v. the United Kingdom, richtungsweisend: hier hat der EGMR erklärt, dass Art. 3 EMRK verletzt sei, wenn ein an AIDS erkrankter Beschwerdeführer in ein Land abgeschoben werde, in dem seine medizinische Versorgung nicht gesichert sei. 
zu etablieren. ${ }^{94}$ Neuntens muss nach der ständigen Rechtsprechung des EGMR, der Staat vorbeugende Maßnahmen zum Schutz der Rechte Einzelner nur ergreifen, wenn insbesondere ,die Behörden wussten oder hätten wissen müssen, dass eine tatsächliche und unmittelbare Gefahr für das Leben einer bestimmten Person oder von mehreren Personen besteht ", ${ }^{95}$ bezahlbare technische Abhilfen existieren ${ }^{96}$ und der Staat eine gewisse Steuerungsmöglichkeit hat. ${ }^{97}$

\section{Schlussbemerkung und Ausblick}

Ziel dieses Artikels war es im Gesamtüberblick aufzuzeigen, dass die Mechanismen des EGMR und insbesondere die von ihm entwickelten positiven Schutzpflichten durchaus auf Klimaschutzfälle anwendbar sind. Auf spezifische klimarelevante Fallkonstellationen, z. B. auf durch Klimawandel verursachte Migration oder Eigentums- und Wohnungsverlust aufgrund von Überflutungen, konnte nicht genauer eingegangen werden.

Die derzeitige umweltrechtliche Rechtsprechung des EGMR basiert im Wesentlichen auf der Prämisse, dass der Staat verantwortlich und haftbar für Menschenrechtsverletzungen in seinem Hoheitsgebiet ist. Die negativen Folgen des Klimawandels jedoch sind eine Folge global verursachter Emissionen und somit nur schwer einem einzelnen Staat zurechenbar. Dies bedeutet jedoch nicht, dass ein einzelner Staat in einem klar umrissenen Bereich nicht die Kontrolle über seine eigenen Politiken haben kann.

Es gibt eine Reihe von potenziell neuen Herausforderungen, mit denen sich der EGMR in den kommenden Jahren möglicherweise befassen muss, darunter Szenarien wie (a) ein herkömmlicher grenzüberschreitender Schaden, d.h. ein Schaden, der hauptsächlich in Staat X verursacht wird, aber auch schädliche Auswirkungen hat im Staat Y; (b) multinationale Unternehmen, die schwerwiegende Klimaschäden verursachen, die wiederum zu Verletzungen der Menschenrechte lokaler Bevölkerungsgruppen führen; c) Klimaflüchtlinge, die aufgrund des Non-Refoulement-Prinzips nicht in ihr Herkunftsland zurückgeführt werden können; (d) Umweltverschmutzungen verursacht durch kollektive globale Umweltverschmutzung wie z.B. Klimawandel. Wenn ein Staat (wirtschaftliche) Aktivitäten auf seinem Hoheitsgebiet, die jedoch außerhalb dieses Territoriums erhebliche Klimaschäden verursachen, nicht ausreichend kontrolliert und ggf. unterbindet, unterliegen die Opfer der dadurch möglicherweise entstehenden Beeinträchtigungen der Zuständigkeit dieses Mitgliedstaates, der sich dann ggf. in Strasbourg vor dem EGMR für im Rahmen dieser Verschmutzungen entstandenen Konventionsverletzungen rechtfertigen muss, auch dann, wenn die Schäden extraterritorial eingetreten sind. Klimapolitik beinhaltet häufig einen Ausgleich zwischen widerstreitenden wirtschaftlichen Interessen. Hier findet derzeit ein Umdenken statt, wie z.B. jüngst der Fall um die Erweiterung des Flughafens Heathrow ${ }^{98}$ gezeigt hat. Anders als noch im Fall Hatton überwogen hier Klimaschutzerwägungen gegenüber wirtschaftlichen Interessen eines Landes. Knackpunkt für etwaige Kläger wird sein, das Kriterium der Opfereigenschaft zu erfüllen. Welche Flexibilität wird der EGMR angesichts der globalen Bedrohungen, die der Klimawandels mit sich bringt, diesem Kriterium künftig entgegenbringen? Inwieweit fühlt sich der EGMR selbst dem Vorsorgeprinzip verpflichtet? In welchen Fällen wird er eine dynamischevolutive Auslegung verfolgen? Wieviel Zeit bleibt für eine vorausschauende Weiterentwicklung der Doktrin der positiven Schutzpflichten und der Anforderung an die Opferqualität in Klimaschutzfällen? Es ist davon auszugehen, dass die Zahl der Klagen vor dem EGMR im Klimabereich weiter zunehmen wird, insbesondere auch vor dem Hintergrund des de facto erweiterten Klagerechts von Verbänden. Auch die Tatsache, dass das Protokoll Nr. 16 der EMRK ${ }^{99}$ nun in Kraft getreten ist, könnte zu einer weiteren Stärkung des EGMR im Bereich des Klimaschutzes führen. Wie bereits in der Vergangenheit, ${ }^{100}$ wird sich der EGMR hier auch von Entwicklungen des Völkerrechts inspirieren lassen.

94) Der z. B. in EGMR, Urt. v. 14.3.2006 - Nr. 23276/04 - Hussein v Albania, angewendete ,Joint-Venure-Ansatz“ könnte hier jedoch Lösungsansätze liefern; es könnte möglicherweise auch argumentiert werden, dass es für die Feststellung einer Konventionsverletzung ausreicht, wenn ein Staat seine Bevölkerung nicht angemessen schützt, indem er z.B. die im Pariser Abkommen genannten Ziele nicht erreicht; s. des Weiteren z.B. Boyle, ,Making the Polluter Pay? Alternatives to State Responsibility in the Allocation of Transboundary Environmental Costs', in: Francioni/Scovazzi (Hrsg.), International Responsibility for Environmental Harm, 1991, 378.

95) EGMR, Urt. v. 28.10.1998 - Nr. 23452/94 - Osman v United Kingdom, $\mathbb{\$} 166$.

96) Besson, Les obligations positives de protection des droits fondamentaux: un essai en dogmatique comparative', Revue de droit suisse, 2003, 122 (1), 49-96.

97) S. dahingehend z.B. EGMR, Urt. v. 9.6.1998 - Nr. 23413/94 - L.C.B. v United Kingdom, $\$ 36 \mathrm{ff}$.

98) S. In den meisten neueren Fällen liegen Interessenskonflikte zwischen dem Recht auf Eigentum und Umweltschutz vor, s.z.B. EGMR, Zulässigkeitsentscheidung v. 6.2.2018 - Calancea and Others $v$ the Republic of Moldova - Nr. 23225/05; EGMR, Urt. v. 6.2.2018 - Nr. 36184/13 - Kristiana Ltd. v Lithuania; EGMR, Urt. v. 7.6.2018 - Nr. 44460/16 - O'Sullivan McCarthy Mussel Development Ltd v Ireland; EGMR, Urt. v. 12.6.2018 - Nr. 70520/10, 21920/10 und 41876/11 Beinarovič and Others v Lithuania; EGMR, Urt. v. 6.9.2018 - Nr. 3401/09 - Dimitar Yordanov v Bulgaria; EGMR, Zulässigkeitsentscheidung v. 2.4.2019 - 54494/11, 67699/11 und 21251/12 - Pop and Others v Romania; EGMR, Zulässigkeitsentscheidung v. 26.11.2019 - Nr. 64863/13 - Yașar v Romania.

99) Dieses Protokoll vom 2.10.2013 (in Kraft seit dem 1.8.2018) sieht vor, dass sich die Verfassungsgerichte bzw. die letztinstanzlichen Gerichte in einem anhängigen Fall mit Fragen zur Auslegung der EMRK für ein Gutachten (,,advisory opinion“) an den EGMR wenden können.

100) EGMR, Urt. v. 27.1.2009 - Tătar v. Rumänien - Nr. 67021/01, $\$ 111$ (hier verweist der EGMR auf die Aarhus-Konvention, obwohl diese von Rumänien zu diesem Zeitpunkt noch nicht ratifiziert war). 